\title{
8 EXERCISE IN OSTEOPOROSIS
}

Shweta Shenoy Secretary General, Indian Association of Sports Medicine, Faculty of Sports, Medicine, Guru Nanak Dev University, Amritsar, Punjab, India

10.1136/bjsm.2010.078725.8

Osteoporosis is a systemic disease characterised by low bone mass and microarchitectural deterioration of bone tissue, with a consequent increase in skeletal fragility and susceptibility to fractures (Consensus Report 1993). Decreased bone mass in the elderly arises because of the degradation of normal balance between bone resorption and bone formation observed in the young adult (Bass et al 1999 and Hansen et al 1991). Pharmacological treatment of osteoporosis entails the use of medications that favourably alter bone remodelling. Current strategies include the administration of antiresorptive agents to inhibit the elevated rate of resorption and the administration of anabolic agents that stimulate osteoblastic bone formation (Mc Dermott et al 2004) options that are extremely limited. Mechanical loading of the bone through the application of exercise has substantial potential to induce bone formation. Yet, studies exploring the use of exercise in humans for the purpose of increasing bone mass have met with mixed results (Turner et al 1994). This article explores the practical considerations for the use of exercise strategies in the enhancement of bone mass. 